\title{
Parameter Sensitivity Analysis of Geotechnical Engineering System Using Neural Network Ensemble
}

\author{
L.X. Pan \\ Department of Engineering Mechanics \\ Hohai University \\ Nanjing, China \\ Y.R. Zhang \\ College of Science \\ Hohai University \\ Nanjing, China
}

\author{
M.S. Cao \\ Department of Engineering Mechanics, \\ Hohai University, \\ Nanjing, China \\ Institute of Fluid-Flow Machinery, \\ Polish Academy of Sciences, \\ Gdansk, Poland \\ D. Novak
}

Institute of Structural Mechanics, Faculty of Civil Engineering Brno University of Technology, Czech Republic

\begin{abstract}
Neural networks-based sensitivity analysis of parameters of geotechnical engineering systems has become a research focus of increasing interest. This study presents a neural network ensemble-based parameter sensitivity analysis method to investigate the sensitivity of variables leading to lateral deformation in an earth-retaining wall of foundation pit system. This method allows not only identification of the dominant variables of the foundation pit system but it also helps to reveal of the relationship between the explicative (input) and dependent (output) variables of the system. The effectiveness of the proposed method is validated by the typical case in geotechnical engineering system.
\end{abstract}

Keywords-parameter sensitivity analysis; neural network ensemble; geotechnical engineering system

\section{INTRODUCTION}

Mathematical models are often utilized to approximate various highly complex engineering, physical, environmental, social, and economic phenomena. One of the steps in model development is the determination of the sensitivities of the parameters by identifying significant and insignificant variables of the system, which provides insight into the cause and effect relationship between the explicative (input) and dependent (output) variables of an analyzed system, conducive to creation of a simplified model for the system.

In recent studies, neural networks have been employed as an alternative of statistical methods to perform sensitivity analysis. In particular, neural network ensemble (NNE) exhibits better performance than traditional statistical methods. NNE is a learning paradigm where a group of neural networks are independently trained to perform the same task [1], which has been used to improve the generalization ability of neural network model. The typical application of NNE was described in [2], where the NNE is applied to perform sensitivity analysis for geotechnical engineering problems with the input perturbation as the sensitivity analysis algorithm. This study uses NNE to deal with sensitivity of variablesleading to lateral deformation in an earth-retaining wall of foundation pit system.

\section{FUNDAMENTALS}

\section{A. Neural Network Modeling}

Neural network modelingis thebasic foundation for neural networks-based parameter sensitivity analysis. One of principal aims of neural network modelingis to achieve the optimal network performance, of which the network is capable of correctly capturing the intrinsic relationship between explicative and dependent variables.

Figure I shows a simple model of back-propagation (BP) neural networks[3]. The number of neurons in the input (first) layer of the neural networksis equal to the number of attributes used as independent variables. The last layer is the network output, where the number of neurons corresponds to the number of variables or output data points to be predicted. The inputs $\left(\left\{P_{1}, \ldots, P_{r}\right\}\right)$ fed in the respective neuron are multiplied by their respective weights $\left(\left\{w_{11}^{1}, \ldots, w_{r 1}^{1}\right\}\right)$ and adding the bias value $\left(b_{1}^{1}\right)$ are used to estimate the outcome from each neuron. Each neuron uses the respective input vectors, the weights and bias as specified in equation (1):

$$
q^{1}=f_{1}\left(\sum_{i=1}^{i=r} P_{i} w_{i 1}^{1}+b_{1}^{1}\right) \quad(1)
$$

which shows an example of how the outcome of hidden node $q^{1}$ is estimated. During learning the weights are adjusted so that the error between the actual and predicted values for the dependent variable is diminished. The backpropagation learning algorithm is usually used to calculate derivatives of performance of the mean square error with respect to the weight and bias variables. 


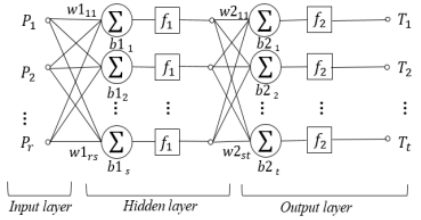

FIGURE I. A SIMPLE MODEL OF BACK-PROPAGATION (BP)NEURAL NETWORKS.

\section{B. Input Perturbation Algorithm}

Sensitivity analysis algorithm is an essential factor for parameter sensitivity analysis. Various sensitivity analysis algorithms can be found in existing studies including partial derivatives algorithm[4], input perturbation algorithm [5-6], connection weights algorithm [7-8], etc., of which the input perturbation algorithmismost widely used. This algorithm produces results of sensitivity analysis based on assessing the effect of small input perturbation in each input on the neural network estimation. The result of sensitivity analysis is yielded by ranking the effect on neural network estimation induced by the same perturbation fashion in every input variable. The input variable whose perturbation influences the output most possesses the highest sensitivity or importance.

In principle, the mean squared error of the neural network estimation increases with the greater degree of perturbation caused by adding noise to the selected input variable. The changes of input variable take the form of $P_{i}=P_{i}+\delta$, where $P_{i}$ is the selected input variable and $\delta$ is noise. $\delta$ increases in steps of $5 \%$ of the mean magnitude of input value up to $15 \%$. According to the increasing magnitude of mean squared error due to each input variable change, the input variables can be ranked, i.e., resulting in a sensitivity analysis outcome.

\section{NNE-BASED PARAMETER SENSITIVITY ANALYSIS}

\section{A. The Property of NNE-Based Parameter Sensitivity Analysis}

In existing studies, a single model of neural networks is commonly employed to perform sensitivity analysis, with better performance than the traditional methods addressed. However, there is a limitation for a single neural networksbased parameter sensitivity analysis, mainly referring to the instability of neural network modeling. This instability influences the accuracy and reliability of neural networksbased parameter sensitivity analysis. In essence, the performance of neural networks-based parameter sensitivity analysis is mainly determined by the trained network structure. However, it is comparatively difficult to explicitly determine a most optimal and uniform network structure [9], which originates from various reasons, such as no reliable theoretical for optimizing the number of hidden layers or number of neurons in each layer [10], the influence of random initialization values[11-12], the ceasing training requires more and less human intervention [13] etc.

To address this limitation, NNE is utilized to tackle the instability of sensitivity analysis relying on a single model of neural networks.

\section{B. Algorithm}

The NNE-based parameter sensitivity analysis strategy involves the following four basic steps: (i) empirically choose excellent types of neural networks as the seeds, which should be particularly specialized and efficient in handling nonlinearity problems; (ii) each seed producesdifferent network forms, each of which performs $\mathrm{k}$ times, to create candidate groups for sensitivity analysis; (iii) from each candidate model group, pick up m neural network models with better performance indicated by less generalization error to form superior neural network ensemble; (iv) calculating the total scores of each explicative variable, then ranking the explicative variables based on the total scores. The smaller the ranking number is, the more sensitive the variable is.

A schematic of the specific procedure of NNE-based parameter sensitivity analysis strategy is presented in Figure II .

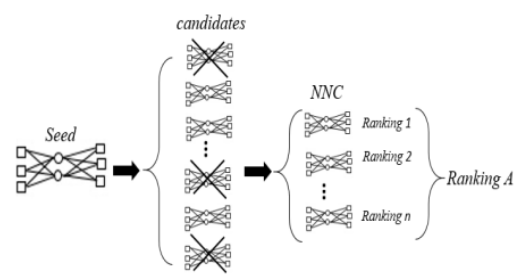

FIGURE II. A SCHEMATIC OF NNE-BASED PARAMETER SENSITIVITY ANALYSIS STRATEGY.

\section{NUMERICAL DEMONSTRATION}

The proposed NNE-based parameter sensitivity analysis is demonstrated in the case of investigating the critical variables influencing the lateral deformation of an earth-retaining wall of foundation pit. This case is built using Algor FEAS software as described in [14]. The description of the case and the result are presented in the rest of this section.

\section{A. Case Description}

The detail messages about the numerical case as follows: the shape of the foundation pit is a polygon, excavation depth $\mathrm{H}=9.71 \mathrm{~m}$; the deep mixing pile ,whose length is $19.0 \mathrm{~m}$ and insertion ratio is approximately 1.0, is used to support safer; the width of the wall is $8.7 \mathrm{~m}$, the inner and the outer layers are inserted bamboo to strengthen. The parameters, influencing on the lateral deformation of earth-retaining wall of foundation pit, need to identify areq, $E, \lambda, C, \phi$, which are arranged by the orthogonal test before simulation. The purpose of the simulation is to obtain the lateral deformation y. The detail data appeared in simulation are shown in Table I . 
TABLE I. THE DETAIL DATA OF SIMULATION.

\begin{tabular}{|c|c|c|c|c|c|c|}
\hline \multirow[t]{2}{*}{ NO. } & 1 & 2 & 3 & 4 & 5 & \multirow{2}{*}{$\begin{array}{c}y \\
\text { (cm) }\end{array}$} \\
\hline & $q(k p a)$ & $E_{(k p a)}$ & $\lambda$ & $C_{(\mathrm{kpa})}$ & $\phi$ & \\
\hline 1 & $1(5.0)$ & $1(3855)$ & $1(0.325)$ & $1(5.63)$ & $1(0.1386)$ & 63.7 \\
\hline 2 & 1 & 2(6168) & $2(0.376)$ & $2(7.44)$ & $2(0.1834)$ & 35.3 \\
\hline 3 & 1 & $3(7710)$ & $3(0.410)$ & $3(8.65)$ & $3(0.2133)$ & 26.7 \\
\hline 4 & 1 & 4(9252) & $4(0.444)$ & $4(9.86)$ & $4(0.2432)$ & 20.9 \\
\hline 5 & 1 & $5(11565)$ & $5(0.478)$ & $5(11.68)$ & $5(0.2731)$ & 12.5 \\
\hline 6 & $2.0(8.0)$ & 1 & 2 & 3 & 4 & 55.8 \\
\hline 7 & 2 & 2 & 3 & 4 & 5 & 32.1 \\
\hline 8 & 2 & 3 & 4 & 5 & 1 & 21.9 \\
\hline 9 & 2 & 4 & 5 & 1 & 2 & 16.3 \\
\hline 10 & 2 & 5 & 1 & 2 & 3 & 25.2 \\
\hline 11 & $3(10.0)$ & 1 & 3 & 5 & 2 & 47.8 \\
\hline 12 & 3 & 2 & 4 & 1 & 3 & 26.1 \\
\hline 13 & 3 & 3 & 5 & 2 & 4 & 16.2 \\
\hline 14 & 3 & 4 & 1 & 3 & 5 & 30.4 \\
\hline 15 & 3 & 5 & 2 & 4 & 1 & 22.1 \\
\hline 16 & $4(12 . .0)$ & 1 & 4 & 2 & 5 & 37.1 \\
\hline 17 & 4 & 2 & 5 & 3 & 1 & 18.0 \\
\hline 18 & 4 & 3 & 1 & 4 & 2 & 34.9 \\
\hline 19 & 4 & 4 & 2 & 5 & 3 & 25.8 \\
\hline 20 & 4 & 5 & 3 & 1 & 4 & 18.9 \\
\hline 21 & $5(15.0)$ & 1 & 5 & 4 & 3 & 25.2 \\
\hline 22 & 5 & 2 & 1 & 5 & 4 & 42.4 \\
\hline 23 & 5 & 3 & 2 & 1 & 5 & 30.1 \\
\hline 24 & 5 & 4 & 3 & 2 & 1 & 22.4 \\
\hline 25 & 5 & 5 & 4 & 3 & 2 & 15.6 \\
\hline
\end{tabular}

The data shown in Table 1 as samples are utilized to train and test NNE for parameter sensitivity analysis. Each sample consists of five explicative variables and one dependent variable, as listed in Table II .

TABLE II. VARIABLES ON PARAMETER SENSITIVITY ANALYSIS.

\begin{tabular}{|c|c|c|}
\hline Variable & Characteristics & Variable \\
\hline$q$ & Surface load & Explicative \\
\hline$E \quad$ of & $\begin{array}{l}\text { Deformation modulus } \\
\text { of soil }\end{array}$ & Explicative \\
\hline$\lambda$ & The Poisson's ratio & Explicative \\
\hline$C$ & Soil cohesion & Explicative \\
\hline$\phi$ & $\begin{array}{l}\text { The internal friction } \\
\text { angle of soil }\end{array}$ & Explicative \\
\hline $\begin{array}{ll}y & \mathrm{~T} \\
\mathrm{de} \\
\mathrm{re} \\
\mathrm{fo}\end{array}$ & $\begin{array}{l}\text { The lateral } \\
\text { leformation of earth- } \\
\text { etaining wall of } \\
\text { oundation pit }\end{array}$ & Dependent \\
\hline
\end{tabular}

B. Results

According to the method described in Section 3.2, the BP neural networks are selected as the seeds; establishing the networks as candidates, each of which with five inputs and one output, then perform each candidate 40 times and rank the parameters after each performing; sorting out three kinds of candidates with good performance as ensemble; calculating the total scores of the each parameter, at last ranking the parameters on the basic of the total scores. The result is presented in TableIII. The smaller the ranking number is, the more influential the parameter is.

From the Table III, it reveals that $E$ is the variable of highest sensitivity, the second is $\lambda$, followed by $C, \phi$ and $q$, as a result of which the $E$ and $\lambda$ are identified the two parameters of most significance. The result is similar to the output presented in [14] and it is coincidence with the engineering practice.

TABLE III. SENSITIVITY ANALYSIS RESULT OF INFLUENTIAL FACTORS.

\begin{tabular}{ccccccc}
\hline $\begin{array}{c}\text { Neural } \\
\text { network } \\
\text { model }\end{array}$ & $\begin{array}{c}\text { Score } \\
\text { and } \\
\text { Ranking }\end{array}$ & $\begin{array}{c}q \\
\text { (kpa) }\end{array}$ & $\begin{array}{c}E \\
\text { (kpa) }\end{array}$ & $\lambda$ & $\begin{array}{c}C \\
\text { (kpa) }\end{array}$ & $\phi$ \\
\hline $\begin{array}{c}\text { Candidate } \\
(1)\end{array}$ & Score & 175 & 43 & 79 & 150 & 153 \\
$\begin{array}{c}\text { Candidate } \\
(2)\end{array}$ & Score & 170 & 44 & 76 & 146 & 164 \\
$\begin{array}{c}\text { Candidate } \\
(3)\end{array}$ & Score & 185 & 40 & 80 & 133 & 162 \\
\hline & $\begin{array}{c}\text { Total } \\
\text { score }\end{array}$ & 530 & 127 & 235 & 429 & 479 \\
& Ranking & 5 & 1 & 2 & 3 & 4 \\
\hline
\end{tabular}

\section{SUMMARY}

To overcome the shortages of the traditional single neural networks, NNE is proposed as an alternative to perform sensitivity analysis of the parameters influencing the lateral deformation of earth-retaining wall of foundation pit. The result shows that $E$ and $\lambda$ are dominating variables and $C$, $\phi$ and $q$ are non-dominating variables, which demonstrates the effectiveness of the NNE-based parameter sensitivity analysis method. The proposed method is an effective way not to identify the parameters, but to confirm the accuracy of numerical simulation results.

\section{ACKNOWLEDGEMENTS}

The authors gratefully acknowledge the financial support provided by the Natural Science Foundation of China (No. 11172091). Last author acknowledge the support of Ministry of Education of Czech Republic under the project KONTAKT No. LH14334.

\section{REFERENCES}

[1] P. Sollich, A. Krogh,"Learning with ensembles: how overfitting can be useful”, In: Touretzky DS, Mozer MC, Hasselmo ME (eds) Advance in neural information processing systems, Denver, CO. MIT Press, Cambridge,Vol.8, pp.190-196, 1996.

[2] M.S. Cao, P.Z. Qiao,"Neural network ensemble-based parameter sensitivity analysis strategy for geotechnical engineering problems”,Neural Computing and Applications,Vol.17(5-6),pp.509-519, 2008.

[3] E. Papatheocharous, H. Papadopoulos and A.S. Andreou, "Feature subset selection for software cost modelling and estimation", Engineering Intelligent,Vol.18, pp.233-246, 2010.

[4] Y. Dimopoulos, P.Bourret, S.Lek, “Use of some sensitivity criteria for choosing networks with good generalization ability”, Neural Processing Letters,Vol.2, pp.1-4, 1995. 
[5] M. Gevrey, I. Dimopoulos, S. Lek,"Review and comparison of methods to study the contribution of variables in artificial neural network models”,Ecological Modelling, Vol.160(3), pp.249-264, 2003.

[6] W. Wang, P. Jones, D. Partridge, "Assessing the impact of input features in a feedforwardneural network", Neural Computing \&Applications, Vol.9(2), pp.101-112, 2000.

[7] G.D. Garson,“Interpreting neural-network connection weights”, Artificial Intelligence Expert, Vol.6, pp.47-51, 1991.

[8] J.D. Olden,D.A. Jackson, “Illuminating the 'black-box': a randomization approach for understanding variable contributions in artificial neural networks”, Ecological Modelling, Vol.154, pp.135-150, 2002.

[9] F. Aires, C. Prigent, W. B. Rossow, "Neural network uncertainty assessment using Bayesian statistics: a remote sensing application”, Neural Computation, Vol.16, pp.2415-2458, 2004.

[10] D. Benaouda, G. Wadge, R.B. Whitmarsh, R.G. Rothwell, C.Macleod, "Inferring the lithology of borehole rocks by applying neural network classification to downwhole logs: an example from the Ocean Drilling Program”, GeophysicalJournal International,Vol.136(2), pp.477491,1999.

[11] A. Atiya, C. Ji, "How initial conditions affect generalization performance in large networks", IEEE Transactions on Neural Networks, Vol.8(2), pp.448-451, 1997.

[12] G. Thimm, E. Fiesler, "High-order and multilayer perception initialization”, IEEE Transactions on Neural Networks,Vol.8(2), pp.349-359, 1997.

[13] S. Natarajan, R.R. Rhinehart, "Automated stopping criteria for neural network training”, In: Proceedings of the American Control Conference, Albuquerque, New Mexico, Vol.4, pp.2409-2413, 1997.

[14] C. Xu, G.B. Ye,"Parameter sensitivity analysis of numerical model by cross test design technique”, Hydrogeology and Engineering Geology (Journal in China), Vol.1,pp.95-97, 2004. 